\title{
Pheromone contents and physiological conditions of adult bean bugs, Riptortus pedestris (Heteroptera: Alydidae), attracted to conspecific males during non- diapause and diapause periods in fields
}

\author{
Nobuo Mizutani, * Tetsuya Yasuda, Takuhiro Yamaguchi and Seiichi MoriYa \\ National Agricultural Research Center (NARC); Tsukuba, Ibaraki 305-8666, Japan \\ (Received 4 October 2007; Accepted 18 February 2008)
}

\begin{abstract}
The contents of five pheromone components and the physiological conditions of wild male and female Riptortus pedestris attracted to bait males during non-diapause (from early July to early September) and diapause (from midSeptember to mid-October) periods in the field were examined. Although tetradecyl isobutyrate (14:iBu), an essential pheromone component, was detected in males attracting conspecific individuals, this component was not detected in almost all males attracted to bait males. This indicated that the attracted males are not attractive to conspecific individuals unlike bait males. During non-diapause, $(E)$-2-hexenyl (E)-2-hexenoate and (E)-2-hexenyl (Z)-3-hexenoate, which are synergistic components of the pheromone, were detected in most males. On the other hand, these components were not detected in most of the males during diapause, indicating that the function of these two components in the attractiveness of males might be related with the sexual communication mechanism. (E)-2-hexenyl hexanoate was detected in almost all males and females attracted to bait males. The physiological conditions were similar between adults attracted to bait males during non-diapause and diapause. Most of the attracted adults had little or no food in their stomachs. This suggested that male-attracted adults of $R$. pedestris are starved and are searching for suitable host plants.
\end{abstract}

Key words: Riptortus pedestris; Riptortus clavatus; aggregation pheromone; physiological condition; diapause

\section{INTRODUCTION}

Adult males of the bean bug, Riptortus pedestris $(=R$. clavatus) (Heteroptera: Alydidae) attract both adult sexes (Numata et al., 1990) and conspecific nymphs (Leal et al., 1995). Tetradecyl isobutyrate (14:iBu), (E)-2-hexenyl (E)-2-hexenoate (E26:E2Hx), (E)-2-hexenyl (Z)-3-hexenoate (E2$6: \mathrm{Z3Hx})$, octadecyl isobutyrate $(18: \mathrm{iBu})$ and $(E)-2-$ hexenyl hexanoate (E2-6:Hx) have been identified as an aggregation pheromone of this bug (Leal et al., 1995; Yasuda et al., 2007a, b). 14:iBu was identified as an essential component, while the four other components have a synergistic effect on $14: \mathrm{iBu}$ in the pheromone system of $R$. pedestris (Mizutani et al., 1997; Endo et al., 2005; Huh et al., 2005; Yasuda et al., 2007a, b).

In $R$. pedestris, wild males and females attracted to males used as attractants have been found to contain E2-6:Hx, a pheromone component (Yasuda et al., 2007b), suggesting that attracted adults (receivers of the pheromone) of $R$. pedestris affect the attractiveness of male adults (releasers of the pheromone). Females of $R$. pedestris used as attractants do not have any pheromone components except for $\mathrm{E} 2-6: \mathrm{Hx}$, and they are not attractive to conspecific individuals by themselves (Mizutani et al., 2007). However, it has not been clarified whether attracted adults have the four pheromone components except for E2-6:Hx.

In $R$. pedestris, feeding and attraction to conspecific individuals are closely related (Morishima et al., 2005), suggesting that the pheromone system of $R$. pedestris plays a key role in food exploitation (Yasuda et al., 2007b). A similar strategy was proposed in the pheromone system of the brownwinged green bug, Plautia crossota stali (P. stali) (Heteroptera: Pentatomidae) (Shiga and Moriya,

* To whom correspondence should be addressed at: E-mail: nobuo@affrc.go.jp DOI: $10.1303 / \mathrm{aez} .2008 .331$ 
1989) and the predatory spined soldier bug, Podisus maculiventris (Heteroptera: Pentatomidae) (Aldrich et al., 1984; Sant'Ana et al., 1997; Shetty and Hough-Goldstein, 1998). This hypothesis regarding the role of pheromones was considered based on the nutritional conditions (the amount of food in their stomach and fat-body development) of adults attracted to males of $P$. crossota stali (Shiga and Moriya, 1989), as well as on the different response to the pheromone source between starved and well-fed adults of $P$. maculiventris (Shetty and Hough-Goldstein, 1998). On the other hand, in regard to the southern green stink bug, Nezara viridula (Heteroptera: Pentatomidae), females in reproductive diapause were unresponsive to males and this was one reason why the maleproduced pheromone is considered to be a sex pheromone (Brennan et al., 1977); however, in $R$. pedestris, the physiological conditions of adults attracted to males used as attractants have not been investigated.

In this paper, we aim to clarify whether adults attracted to males used as attractants contain pheromone components, and to clarify the conditions of the internal organs of attracted male and female adults during non-diapause and diapause periods. In addition, we discuss the role of the composition of each pheromone and its functions in the male-mediated aggregation of $R$. pedestris.

\section{MATERIALS AND METHODS}

Males used as attractants. $R$. pedestris adults were collected from fields in Tsukuba and reared in the laboratory on soybean seeds, red clover (Trifolium pretense) seeds, and water and maintained at $25^{\circ} \mathrm{C}$ with a photoperiod of $16 \mathrm{~L} 8 \mathrm{D}$. Newly emerged adult males were separated into different cages, and virgin males (12-14 days old) were used as attractants in each experiment.

Collection of attracted males and females. A male was put into a stainless steel-mesh cage $(10 \mathrm{~cm}$ outside diameter $\times 8 \mathrm{~cm}$ height $)$ with dried soybean seed and water. The soybean seed was partly covered with aluminum foil to prevent sucking by individuals outside the cage. The cage was fixed in front of a plastic plate $(18 \times 25.5 \mathrm{~cm})$ at a height of ca. $1 \mathrm{~m}$ from the ground (see Mizutani et al., 2007). Ten or twelve traps were placed at approximately $10 \mathrm{~m}$ intervals in a grassy field con- taining rows of coniferous trees at the National Agricultural Research Center (NARC) in Tsukuba, Japan. The traps were set at 1800 , and observations were started at 0900 the next day. Male and female adults on the cage and plate were collected at 0900, 1300, and 1700 each day. The traps were set for three days in each experiment.

Males and females were collected from early July to mid-October 2006. $R$. pedestris exhibit a facultative adult diapause and the critical daylength for the induction of diapause was $13.5 \mathrm{~h}$ at $25^{\circ} \mathrm{C}$ (Numata and Hidaka, 1982). In Tsukuba, the daylength becomes shorter than $13.5 \mathrm{~h}$ around 10 September, and it is considered that $R$. pedestris adults enter diapause in mid-September. Therefore, the collected adults were classified into two groups: adults collected between early July and early September (adults collected during non-diapause), and adults collected between mid-September and mid-October (adults collected during diapause).

Analyses of pheromone components from whole-body extracts of attracted males and females. Intact male or female $R$. pedestris attracted to males used as attractants were individually dipped in $2 \mathrm{ml}$ of hexane for $1 \mathrm{~h}$ at room temperature. The hexane solution of hexadecane $(2 \mu \mathrm{g})$ was added as an internal standard in each sample. The extract was decanted from the male or female body into a glass vial. The residual body was rinsed with $1 \mathrm{ml}$ of hexane, and the rinse was added to the extract. The extracts were stored at $-20^{\circ} \mathrm{C}$ until gas chromatography-mass spectrometry (GC-MS) analysis. The extracts of hexane solution were concentrated to ca. $100 \mu \mathrm{l}$ volumes just before GC-MS analysis.

Quantitative GC-MS analyses were performed using an Agilent 6890N GC with an HP-INNOWax column $(30 \mathrm{~m} \times 0.25 \mathrm{~mm}$ inside diameter $\times 0.25 \mu \mathrm{m}$ film thickness) by splitless injection combined with an Agilent 5975iNetwork Mass Selective Detector using an internal-standard method. Injection was made in the splitless mode in the split/splitless injector using an Agilent 7688 series automatic liquid sampler, with the injector temperature being $230^{\circ} \mathrm{C}$. Helium was used as the carrier gas and the flow rate was $1.0 \mathrm{ml} / \mathrm{min}$ in the constant flow mode. The initial GC oven temperature was $50^{\circ} \mathrm{C}(1 \mathrm{~min}$ hold), increased to $180^{\circ} \mathrm{C}$ at $10^{\circ} \mathrm{C} \mathrm{min}^{-1}$, increased again to $240^{\circ} \mathrm{C}$ at $20^{\circ} \mathrm{C} \mathrm{min}^{-1}$, and was then held 


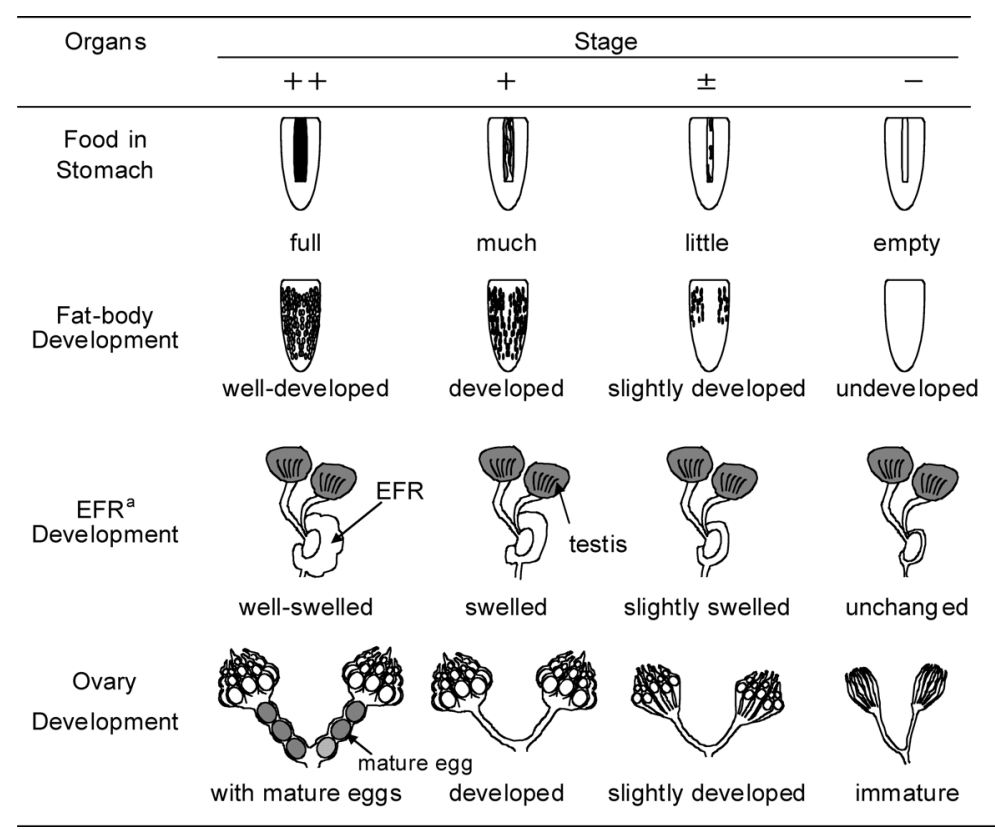

Fig. 1. Classification of conditions of several internal organs of Riptortus pedestris adults. The characteristics of the organs were observed by dissection. ${ }^{\mathrm{a}}$ EFR: erection fluid reservoir.

for $5 \mathrm{~min}$.

Authentic chemicals for quantitative GC-MS analyses. 14:iBu and 18:iBu were obtained from 1tetradecanol and 1-octadecanol, respectively, reacted with isobutyric anhydride and pyridine. E26:E2Hx and $\mathrm{E} 2-6: \mathrm{Z3Hx}$ were obtained from the Fuji Flavor Co. Ltd., and E2-6:Hx and hexadecane were purchased from the Tokyo Chemical Industry Co., Ltd., Japan.

Conditions of internal organs of attracted males and females. After extraction, conditions of the internal organs, such as food in the stomach, fat-body development, and erection fluid reservoir (EFR; male) or ovary (female) development, were observed by dissection of $R$. pedestris adults attracted to males used as attractants. The conditions of internal organs were classified into four stages (Fig. 1). In females, mating status was classified into three stages by the amount of sperm found in the spermatheca $(++$, much sperm; + , little sperm; -, no sperm).

To compare the nutritional condition of adults attracted to bait males with those of adults inhabiting soybean fields, adults inhabiting soybean fields were collected from 31 August to 5 September (during non-diapause, female, 29; male, 39) and from 21 to 22 September (during diapause, female, 41 ; male, 31). The amount of food in the stomach and degree of fat-body development of the collected female and male adults were classified in the same way as described below. The degree of development of the internal organs of the collected adults was compared with those attracted to bait males from 30 August to 1 September (during nondiapause, female, 26; male, 11) and from 19 to 21 September (during diapause, female, 31; male, 39).

\section{RESULTS}

Content of pheromone components from the whole-body extract of attracted males during non-diapause

Seventy-four males were attracted to attracting males during non-diapause in the field. Hexane extracts of the attracted males were individually analyzed using GC-MS. The contents of the pheromone components in each extract are shown in Fig. 2. The individual numbers in Fig. 2 are the collection date and identification number of the extracts (e.g. "0711-M1" means the extract of first male collected in 11 July).

In many extracts (63 of 74), 14:iBu, which is the pheromone's essential component, was not detected. In 11 extracts (Nos. 0711-M2, 0726-M7, 0727-M4，0808-M1，0819-M1，0821-M5，0821M8, 0821-M10, 0821-M13, 0830-M6, and 0830- 

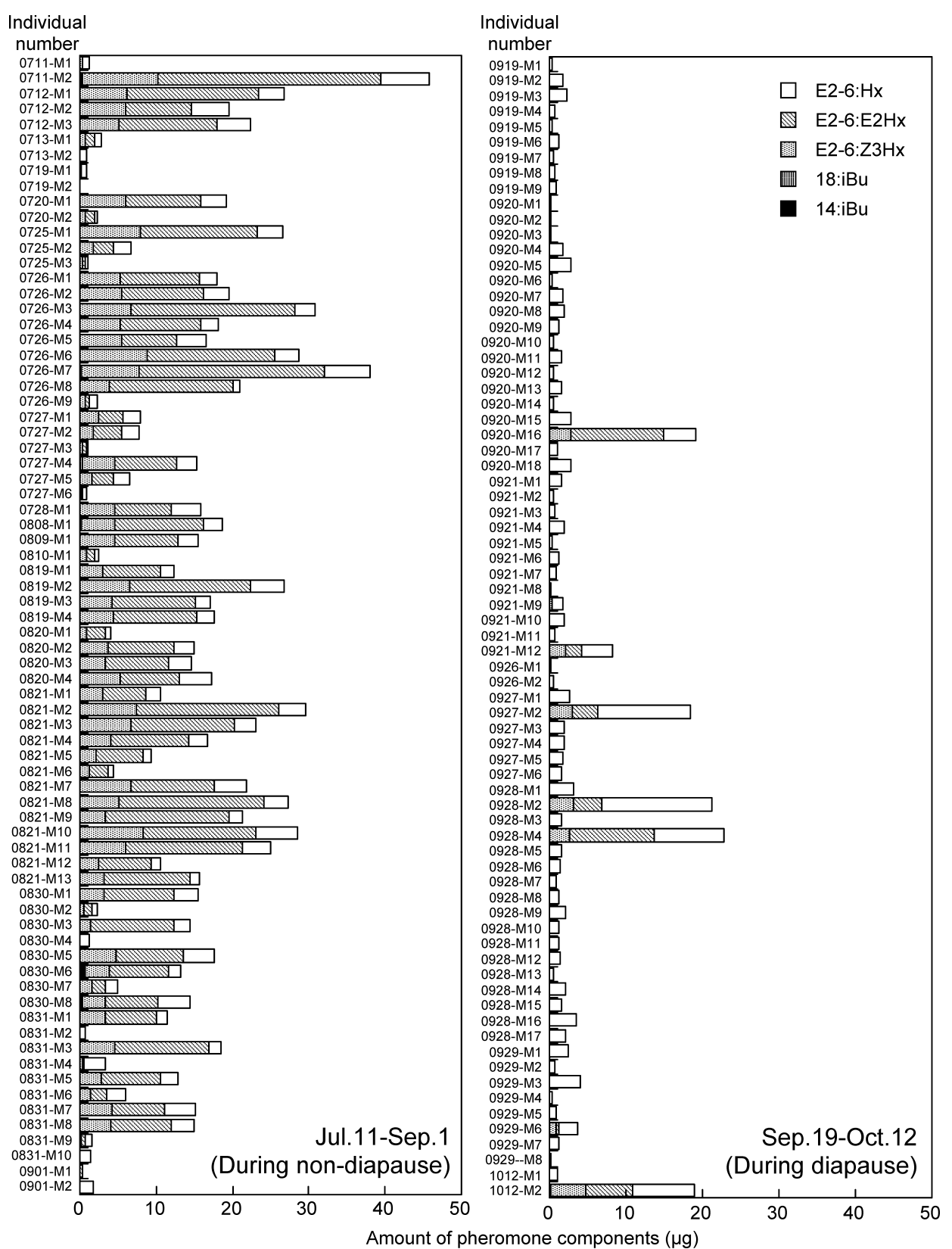

Fig. 2. Individual amounts of pheromone components of whole-body extracts of attracted $R$. pedestris males in the field in Tsukuba in 2006.

M8), 14:iBu was detected. The 14:iBu content in the extracts was $0.09 \pm 0.04 \mu \mathrm{g}$ (mean $\pm \mathrm{SE}) .18: \mathrm{iBu}$ was not detected in 62 of 74 extracts, while it was detected in the other 12 (Nos. 0711-M2, 0713-M2, 0726-M7，0726-M8，0727-M4，0808-M1，0821M5, 0821-M10, 0821-M13，0830-M3，0830-M6, and $0830-\mathrm{M} 8$ ). The $18: \mathrm{iBu}$ content in the extracts was $0.11 \pm 0.03 \mu \mathrm{g}$ (mean $\pm \mathrm{SE}$ ).

E2-6:E2Hx and/or E2-6:Z3Hx were detected in many extracts (67 of 74) (Fig. 2). E2-6:E2Hx and E2-6:Z3Hx contents in the extracts were $8.41 \pm 0.72$ and $3.78 \pm 0.30 \mu \mathrm{g}$ (mean $\pm \mathrm{SE})$, respectively. In 5 extracts (Nos. 0713-M2, 0719-M1, 0830-M4, 0831-M2, and 0901-M2), only small amounts of E2-6:E2Hx and/or E2-6:Z3Hx were detected $(0.1 \mu \mathrm{g}$ or less), and in two extracts (0719M2, 0831-M10), E2-6:E2Hx and E2-6:Z3Hx were not detected at all.

E2-6:Hx was detected in all extracts of attracted males (73 of 74) except in No. 0719-M2. 
Content of pheromone components from the whole-body extract of attracted males during diapause

Sixty-six males were attracted during diapause in the field. $14: \mathrm{iBu}$ and $18: \mathrm{iBu}$ were detected in only one extract (1012-M2, 14:iBu, $0.09 \mu \mathrm{g}$; $18: \mathrm{iBu}, 0.11 \mu \mathrm{g})$. In 52 of 66 extracts, E2-6:E2Hx and E2-6:Z3Hx were not detected (Fig. 2). In seven extracts (Nos. 0920-M16, 0921-M9, 0921-M12, 0927-M2, 0928-M2, 0928-M4, and 1012-M2), E26:E2Hx and/or E2-6:Z3Hx were detected. E2$6: \mathrm{E} 2 \mathrm{Hx}$ and $\mathrm{E} 2-6: \mathrm{Z} 3 \mathrm{Hx}$ contents in the extracts were $5.49 \pm 1.71$ and $2.67 \pm 0.47 \mu \mathrm{g}$ (mean $\pm \mathrm{SE}$ ), respectively. In seven extracts (Nos. 0919-M4, 0920-M5, 0920-M18, 0921-M10, 0928-M1, 0928$\mathrm{M} 12$, and 1012-M1), E2-6:E2Hx and/or E2$6: \mathrm{Z} 3 \mathrm{Hx}$ were detected only slightly $(0.1 \mu \mathrm{g}$ or less).

E2-6:Hx was detected in all extracts of attracted males, while only E2-6:Hx was detected in many extracts of attracted males collected during diapause (52 of 66).

\section{Content of pheromone components from whole- body extracts of attracted females}

Fifty-one females (during non-diapause) and 66 females (during diapause) were attracted to bait males in the field. The collected females were not classified into two groups, because the proportions of the pheromone components of females attracted to bait males, did not differ between females attracted during diapause and non-diapause. Hexane extracts of 117 females attracted to male adults used as attractants in the field were separately analyzed using GC-MS. 14:iBu, 18:iBu, and E2$6: \mathrm{Z} 3 \mathrm{Hx}$ were not detected in these extracts, while E2-6:E2Hx was detected only in slight amounts (below $0.06 \mu \mathrm{g}$ ) in nine of the extracts. E2-6:Hx was detected in all extracts, with the E2-6:Hx content varying widely among them.

\section{Conditions of internal organs of attracted males and females}

Sixty-eight percent (during non-diapause) and $88 \%$ (during diapause) of attracted males had little or no food in their stomachs (Fig. 3), and almost $100 \%$ (during diapause) and $75 \%$ (during non-diapause) of attracted females had less food in their stomachs (Fig. 3). Forty-five percent (during nondiapause) and 52\% (during diapause) of attracted males and 49\% (during non-diapause) and 64\% (during diapause) of attracted females showed an undeveloped or slightly developed fat-body (Fig. 3).

Sixty-nine percent of males collected during non-diapause had developed EFR (Fig. 3). On the other hand, $91 \%$ of males collected during diapause had slightly swollen or unchanged EFR. Similarly, $63 \%$ of females collected during nondiapause had developed or mature ovaries, while almost all females collected during diapause had slightly developed or immature ovaries (Fig. 3).

Seventy-eight percent of females collected during non-diapause had sperm in the spermatheca. Ninety-four percent of females collected during diapause had no sperm in the spermatheca (Fig. 3).

The amount of food in the stomach and the degree of fat-body development were similar between male and female adults. Adults attracted to bait males had significantly less food in their stomachs than adults collected in soybean fields both at nondiapause and diapause (Fig. 4) (chi-square test, $p<0.01$ ). With regard to fat-body development, there were no significant differences between attracted and collected adults.

\section{Relationship between conditions of internal or- gans and amount of pheromone components in attracted males and females}

In males collected during non-diapause $(n=74)$, the amounts of three pheromone components (E26:E2Hx, E2-6:Z3Hx, and E2-6:Hx) in whole-body extracts of males were compared with the condition of their internal organs (food in stomach, fatbody development, and EFR development) (Fig. 5). Males with well-developed organs showed a tendency to have larger amounts of E2-6:E2Hx, E26:Z3Hx, and E2-6:Hx than males with undeveloped organs, and significant differences were seen in the amounts of components among stages in some combinations of organs and compositions (e.g., E2-6:Hx-food in stomach etc.) (Mann-Whitney $U$-test with Bonferroni correction after Kruskal-Wallis test, $p<0.05$ ).

In females collected during non-diapause $(n=51)$, the amounts of E2-6:Hx in the wholebody extracts were compared with the condition of their internal organs (food in stomach, fat-body development, and ovary development), respectively (Fig. 6). Females with developed organs showed a 

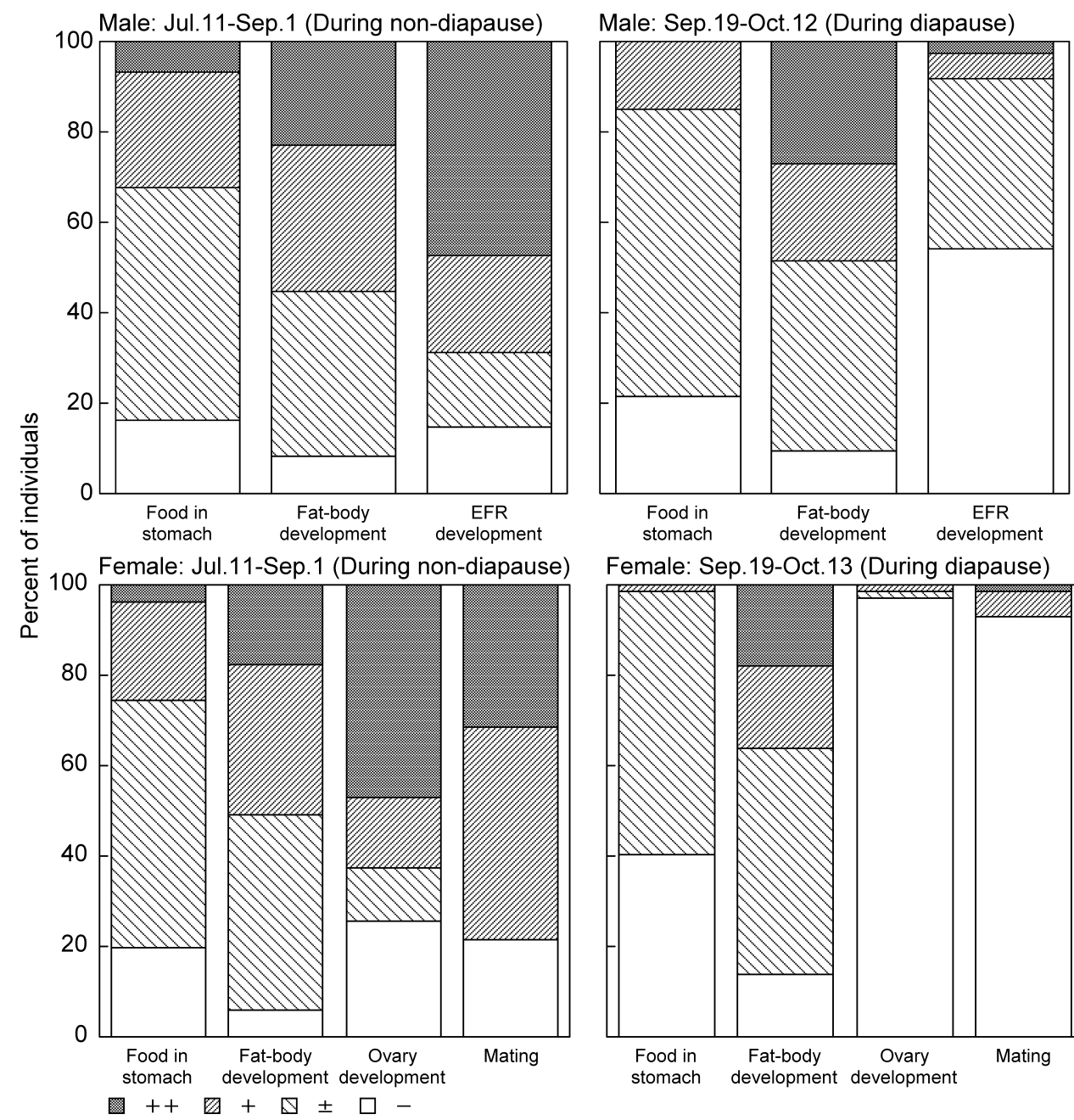

Female: Sep.19-Oct.13 (During diapause)

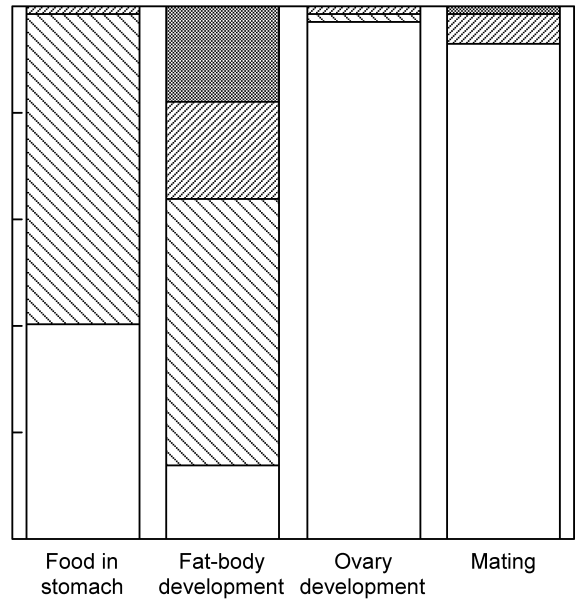

Fig. 3. Conditions of internal organs (amount of food in stomach, degree of fat-body development, and degree of erection fluid reservoir development, degree of ovary development, and mating status) of attracted males and females of $R$. pedestris in the field in Tsukuba in 2006.

tendency toward larger amounts of E2-6:Hx than females with undeveloped organs; however, this tendency in females was not so clear as in males.

\section{DISCUSSION}

Males attracting conspecific adults and nymphs contained 14:iBu (Mizutani et al., 2007). In the present study, males used as attractants were determined to contain 14:iBu by GC-MS analysis; however, most of the males attracted to bait males during non-diapause and all males attracted to bait males during diapause did not contain $14: \mathrm{iBu}$, which is an essential component of the $R$. pedestris pheromone. The present result indicates that attracted males are not attractive to conspecific indi- viduals by themselves.

Attracted males had only synergistic components (E2-6:E2Hx, E2-6:Z3Hx, and E2-6:Hx; Endo et al., 2005; Huh et al., 2005) without the essential component of the pheromone, 14:iBu. Furthermore, the proportions of males with E2-6:E2Hx and E2-6:Z3Hx differed between males attracted during diapause and non-diapause. These components were detected in many extracts of males collected during non-diapause, while they were hardly found in extracts of those collected during diapause. Males with undeveloped EFR showed a tendency toward a lower amount of E2-6:E2Hx and E2-6:Z3Hx than males with well-developed EFR. These results indicate that sexually immature males do not have E2-6:E2Hx and E2-6:Z3Hx. In 

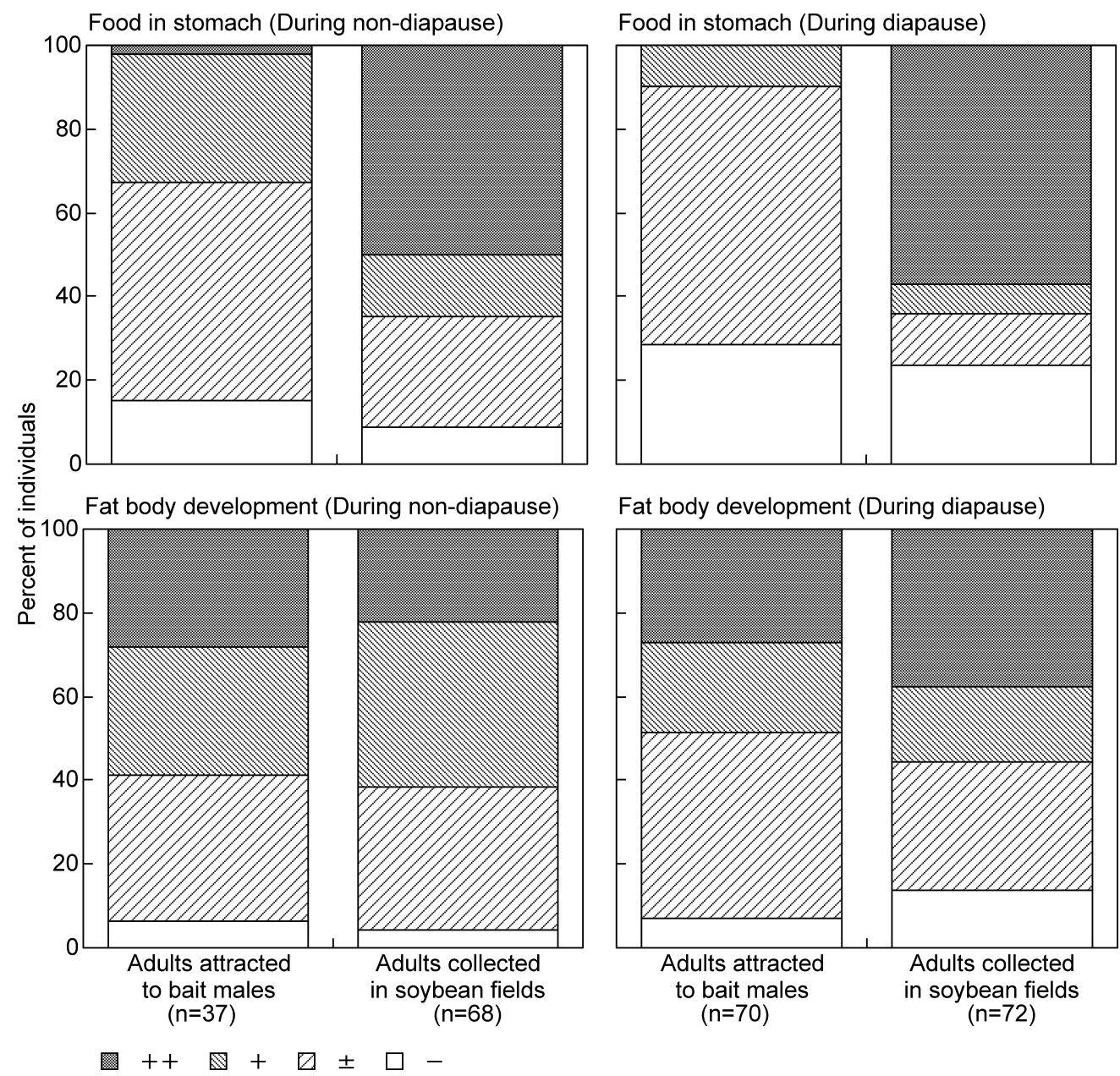

Fat body development (During diapause)

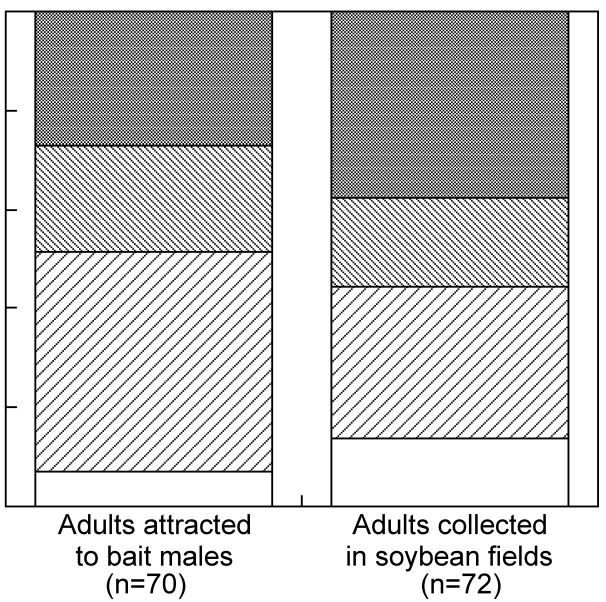

Fig. 4. Nutritional conditions (amount of food in the stomach and degree of fat-body development) of $R$. pedestris adults in the field in Tsukuba in 2006.
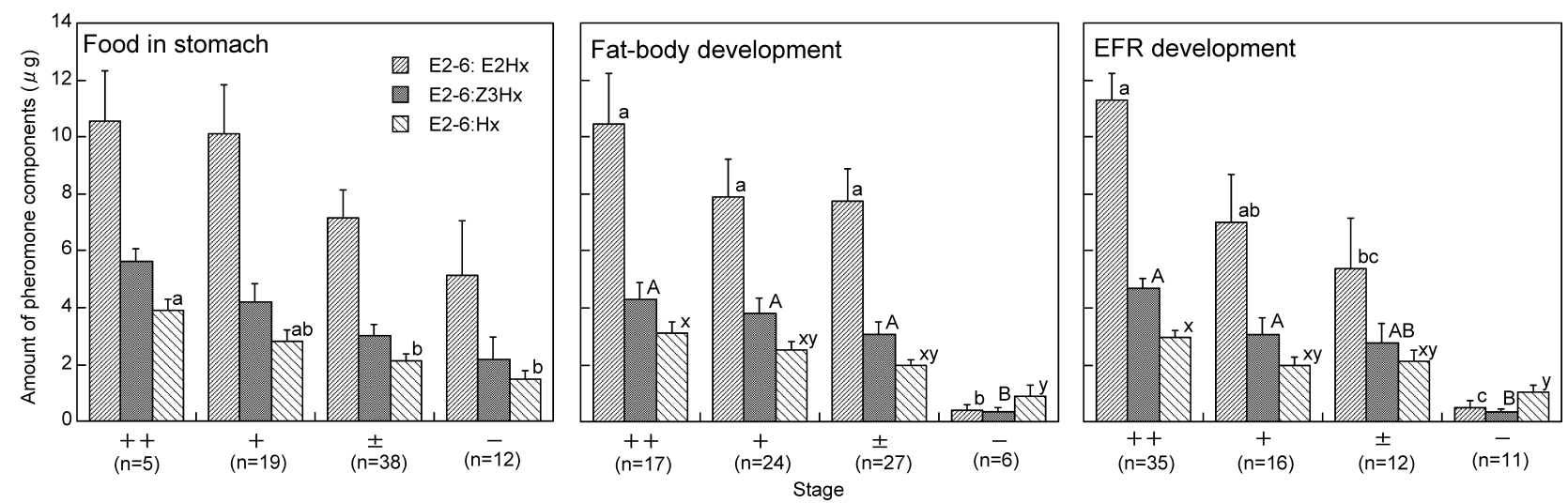

Fig. 5. Relationship between the conditions of internal organs (amount of food in stomach, degree of fat-body development, and degree of erection fluid reservoir development) and the content of pheromone components (E2-6:E2Hx, E2-6:Z3Hx, and E26:Hx) of whole-body extracts of attracted $R$. pedestris males in Tsukuba during non-diapause in 2006. 

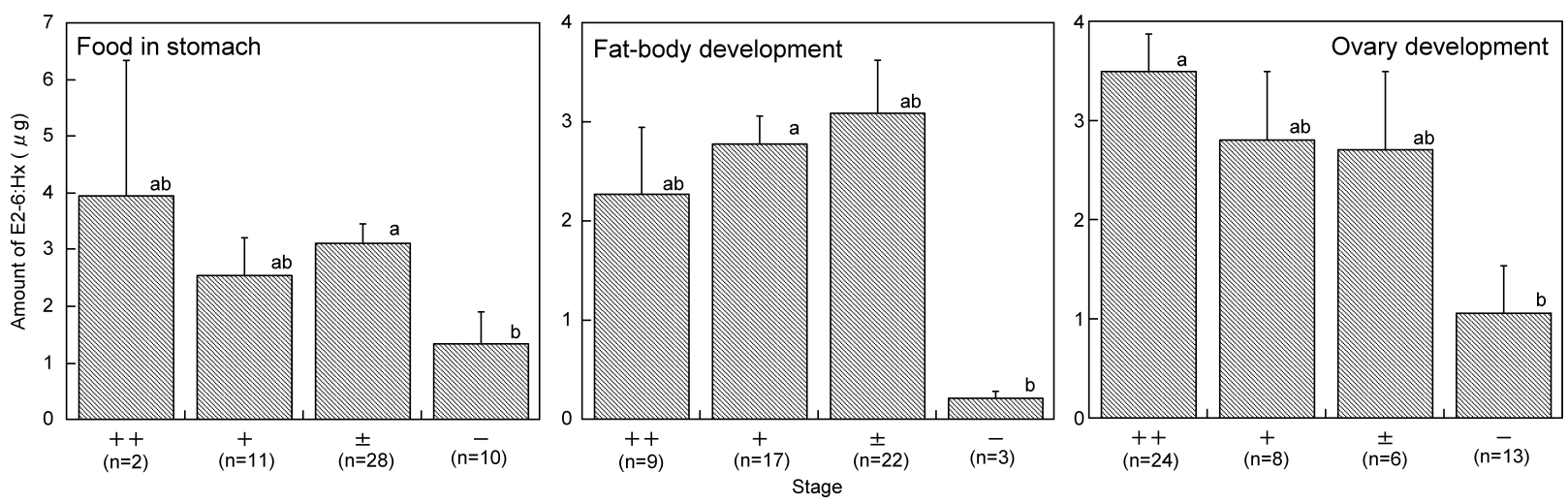

Fig. 6. Relationship between the conditions of internal organs (amount of food in stomach, degree of fat-body development, and degree of ovary development) and the amount of E2-6:Hx of whole-body extracts of attracted R. pedestris females in Tsukuba during non-diapause in 2006. In 123 extracts from females, only E2-6:Hx was detected; other components (18:iBu, E2-6:E2Hx, and $\mathrm{E} 2-6: \mathrm{Z3Hx}$ ) were detected in only negligible amounts.

N. viridula, diapausing males were found to be much less attractive to females than non-diapausing males in terms of pheromone communication (Brennan et al., 1977). The fact that only sexually mature males of $R$. pedestris have $\mathrm{E} 2-6: \mathrm{E} 2 \mathrm{Hx}$ and/or E2-6:Z3Hx suggests that the function of E2$6: \mathrm{E} 2 \mathrm{Hx}$ and $\mathrm{E} 2-6: \mathrm{Z3Hx}$ in the attractiveness of males should be related to their sexual communication mechanism.

Both attracted males and females had less food in their stomachs, with females having far less food in their stomachs than males. Attracted females and males starved compared with those collected in soybean fields. In $P$. crossota stali, male-attracted adults were considered to have few opportunities to ingest suitable foods and to disperse in search of suitable food plants, because adults had far less food in their stomach and showed less developed fat-bodies in comparison with those feeding on suitable food plants (Shiga and Moriya, 1989). The results of this study suggest that maleattracted adults of $R$. pedestris are also starved and in search of suitable food plants. In addition, the present results support the hypothesis that $R$. pedestris adults, especially females, use the pheromone as a cue indicating the presence of food.

Attracted males that stored plentiful nutritional reserves showed a tendency to have a larger amount of E2-6:E2Hx, E2-6:Z3Hx, and E2-6:Hx than males that stored only poor nutritional reserves. In males used as attractants in field experiments, males having well-developed fat-bodies showed a tendency to have more $14: \mathrm{iBu}$ than those with undeveloped fat-bodies (Mizutani et al., 2007). These results indicate that the production of pheromone components including $14: \mathrm{iBu}$ and the nutritional condition of males are closely related in $R$. pedestris. Further detailed experiments should be performed to clarify the relationship between the release of the pheromone and the timing of feeding in males.

\section{REFERENCES}

Aldrich, J. R., J. P. Kochansky and C. B. Abrams (1984) Attractant for a beneficial insect and its parasitoids: pheromone of the predatory spined soldier bug, Podisus maculiventris (Hemiptera: Pentatomidae). Environ. Entomol. 13: 1031-1036.

Brennan, B. M., F. Chang and W. C. Mitchell (1977) Physiological effects on sex pheromone communication in the southern green stink bug, Nezara viridula. Environ. Entomol. 6: 169-173.

Endo, N., T. Wada, N. Mizutani, S. Moriya and R. Sasaki (2005) Ambiguous response of Riptortus clavatus (Heteroptera: Alydidae) to different blends of its aggregation pheromone components. Appl. Entomol. Zool. 40: 41-45.

Huh, H. S., K. H. Park, W. D. Seo and C. G. Park (2005) Interaction of aggregation pheromone components of the bean bug, Riptortus clavatus (Thunberg) (Heteroptera: Alydidae). Appl. Entomol. Zool. 40: 643-648.

Leal, W. S., H. Higuchi, N. Mizutani, H. Nakamori, T. Kadosawa and M. Ono (1995) Multifunctional communication in Riptortus clavatus (Heteroptera: Alydidae): conspecific nymphs and egg parasitoid Ooencyrtus nezarae use the same adult attractant pheromone as chemical cue. J. Chem. Ecol. 21: 973-985.

Mizutani, N., T. Wada, H. Higuchi, M. Ono and W. S. Leal (1997) A component of a synthetic aggregation 
pheromone of Riptortus clavatus (Thunberg) (Heteroptera: Alydidae), that attracts an egg parasitoid, Ooencyrtus nezarae (Hymenoptera: Encyrtidae). Appl. Entomol. Zool. 32: 504-507.

Mizutani, N., T. Yasuda, T. Yamaguchi and S. Moriya (2007) Individual variation in the amounts of pheromone components in the male bean bug, Riptortus pedestris (Heteroptera: Alydidae) and its attractiveness to the same species. Appl. Entomol. Zool. 42: 629-636.

Morishima, M., K. Tabuchi, K. Ito, N. Mizutani and S. Moriya (2005) Effect of feeding on the attractiveness of Riptortus clavatus (Thunberg) (Heteroptera: Alydidae) males to conspecific individuals. Jpn. J. Appl. Entomol. Zool. 49: 262-265 (in Japanese with English summary).

Numata, H. and T. Hidaka (1982) Photoperiodic control of adult diapause in the bean bug, Riptortus clavatus Thunberg (Heteroptera: Coreidae) I. Reversible induction and termination of diapause. Appl. Entomol. Zool. 17: $530-538$.

Numata, H., M. Kon and T. Hidaka (1990) Male adults attract conspecific adults in the bean bug, Riptortus clavatus Thunberg (Heteroptera: Alydidae). Appl. Entomol. Zool. 25: 144-145.
Sant'Ana, J., R. Bruni, A. A. Abdul-Baki and J. R. Aldrich (1997) Pheromone-induced movement of nymphs of the predator, Podisus maculiventris (Heteroptera: Pentatomidae). Biol. Control 10: 123-128.

Shetty, P. N. and J. A. Hough-Goldstein (1998) Behavioral response of Podisus maculiventris (Hemiptera: Pentatomidae) to its synthetic pheromone. J. Entomol. Sci. 33: 72-81.

Shiga, M. and S. Moriya (1989) Temporal and spatial differences in the conditions of the internal organs of adults of the brown-winged green bug, Plautia stali Scott (Heteroptera: Pentatomidae). Bull. Fruit Tree Res. Stn. A16: 133-168 (in Japanese with English summary).

Yasuda, T., N. Mizutani, N. Endo, T. Fukuda, T. Matsuyama, K. Ito, S. Moriya and R. Sasaki (2007a) A new component of attractive aggregation pheromone in the bean bug, Riptortus clavatus (Thunberg) (Heteroptera: Alydidae). Appl. Entomol. Zool. 42: 1-7.

Yasuda, T., N. Mizutani, Y. Honda, N. Endo, T. Yamaguchi, S. Moriya, T. Fukuda and R. Sasaki (2007b) A supplemental component of aggregation attractant pheromone in the bean bug Riptortus clavatus (Thunberg), related to food exploitation. Appl. Entomol. Zool. 42: 161-166. 